\title{
Complexity of magnetic structures: Flares and cycle phase dependence
}

\begin{abstract}
N. Meunier ${ }^{\star}$
Observatoire Midi-Pyrénées, 57 avenue d'Azereix, BP 826, 65008 Tarbes Cedex, France

Received 2 July 2003 / Accepted 17 February 2004

Abstract. A long data set of MDI/SOHO full-disk magnetograms is analyzed to provide a large sample of active regions. The objective is to study the variations of the fractal dimension of these structures on different time scales: in relation to their activity level (spots, flares) and during the solar cycle. First, the variations of the fractal dimension of the structure during solar flares are studied using a very large sample. Variations are found to be of very small amplitude. The complexity of the structures is slightly larger after the flare when considering regions close to the flare $\left(2-3^{\circ}\right)$, while a decrease seems to be observed at larger scales (up to $10^{\circ}$ ), although this should be confirmed using other approaches. The results obtained by Meunier (1999a) concerning the fractal dimension versus the size of the structures for various magnetic thresholds are confirmed on a much larger sample, with a peculiar behavior seen around $550-800 \mathrm{Mm}^{2}$. This scale is observed to increase from cycle minimum to cycle maximum. In addition, I observe that the fractal dimension of active regions reaches a plateau above $3000 \mathrm{Mm}^{2}$ and that structures of a given size are more complex when they have spots. The fractal dimension is strongly variable during the solar cycle, with the sign of the variation depending on their size and on their flux content (spot or no spot) and with an amplitude up to 0.17 between the ascending phase and cycle maximum.
\end{abstract}

Key words. Sun: magnetic fields - Sun: photosphere - Sun: activity - Sun: faculae, plages

\section{Introduction}

The variation of the magnetic topology before, during and after solar flares has been the subject of many studies, because the evolution of the region topology before the flare and the mechanisms for triggering the flare are not well understood yet. Many detailed studies of a given region have been performed using vector magnetograms and extrapolations of photospheric magnetic fields to look at the evolution of the 3D topology (see among others the recent papers by Bleybel et al. 2002; Zhang \& Wang 2002; Fletcher et al. 2001; Zhang \& Wang 2000; Aulanier et al. 2000; Mathew \& Ambastha 2000, and references therein). Some statistical studies of small data sets (no more than a few regions) have also been performed by several authors to study the variation of the complexity of active region during solar flares. A first approach consisted of comparing the complexity of quiet region with flaring region. For example, Patty \& Hagyard (1986) observed that the maximum line-of-sight (hereafter LOS) magnetic field and its gradient were much larger in flaring regions than in quiet regions. Abramenko et al. (2002) studied the structure function exponent on 8 active regions of different flaring activity levels and found a larger degree of intermittency in regions that were more active. Recently, a second approach consisted of studying the variation of the complexity during the evolution of the region before and after flares. This was done by Adams et al. (1997)

* e-mail: meunier@bagn.obs-mip.fr for example, who applied the Hurst analysis to 10 vector magnetograms of an active region and did not observe any variation due to flares, except for the shear of the region: they obtained an increase of the complexity of the region before the flare, and a decrease during the 5 min preceeding the flare. Stark et al. (1997) tested two other methods with the same purpose and showed that they were not very sensitive. On the other hand, Hagyard et al. (1999) studied the vector magnetic field of an active region during $6 \mathrm{~h}$ and found the azimuth to be very variable. Yurchyshyn et al. (2000) and more recently Sorriso-Valvo et al. (2003) computed the cancellation exponent on the helicity for 5 regions. The cancellation exponenent is anti-correlated with the fractal dimension (see Sorriso-Valvo et al. 2003) and it shows a strong increase in complexity at the time of the flare (or during the $3 \mathrm{~h}$ preceeding the flare).

Vector magnetic fields are very useful because the LOS magnetic field is not necessarily the component that is expected to change the most during flares (see Adams 1997 for example). Nothing conclusive concerning LOS fields has been obtained, while recent observations of vector magnetic fields suggest larger variations of physical parameters other than the LOS magnetic field. However, time series of vector magnetograms are scarce. On the other hand, no study of a very large sample of LOS magnetograms has been performed, so it will be interesting to check the variations of the fractal dimension of regions observed that way when a large sample becomes available. This investigation is the first objective of this paper. 
The second objective is to determine the variation of the complexity of active regions on a much longer time scale. The distribution of solar magnetic fields in the photosphere is controled by the generation processes, including the solar dynamo. Statistical analyses such as studies of the size distribution of active regions or of the fractal dimension are necessary to compare the very complex observed regions and models. No realistic models of active regions have been developed to my knowledge so far, although the approach followed by Wentzel \& Seiden (1994), Seiden \& Wentzel (1996) and Vlahos et al. (2002) should provide some clues about the various processes at play in their formation and disappearance. Hopefully they will become more realistic in the near future. Up to now, only very small series of high resolution magnetograms have been analyzed (Ruzmaikin et al. 1991; Balke et al. 1993) or larger sets of full disk images covering a small period (Nesme-Ribes et al. 1996, studying spectroheliograms, Meunier 1999a, studying MDI full-disk magnetograms). Although these studies are obviously related to the solar dynamo, no studies have been performed to study the cycle dependence of the fractal dimension of active regions. I am not aware of analyses of the fractal dimension as a function of flux content either. Therefore, in this Paper I continue the work of Meunier (1999a) on a much longer data set. The study of a long series of LOS MDI magnetograms is possible covering a period from the cycle minimum to the end of the cycle maximum. The main advantage is the huge quantity of data of constant quality, allowing a very good statistical study.

The complexity of magnetic structures, studied using fractal dimension techniques, is primarily related to the complexity of their morphology. However, this complexity must tell us something about the physics underlying the active region and the flares: the turbulent character of the convection means that the magnetic fields generated by the dynamo are intermittent, with a complex structure, and the analysis of their selfsimilarity using fractal dimensions is therefore relevant (see for example Tao et al. 1995 and Vainshtein et al. 1996). This has also been studied for example by Zelenyi \& Milovanov (1991), who have developed a fractal model of sunspots. The study of how this complexity depends on the activity level on various scales should therefore provide clues about the influence of this activity level on the formation processes (for example how the clustering of flux tubes can form large coherent regions) or about the later stages of evolution of the region (processes at play during flares or during the dilution of the active region and spreading of its flux across the solar surface, for example through a percolation process such as the one studied by Balke et al. 1993).

Therefore my objective is two-fold: 1) study of the variation of the fractal dimension from LOS magnetograms during solar flares as well as a detailed analysis of the fractal dimension with magnetic properties of the regions; 2) study of the solar cycle dependence. After describing the observations in Sect. 2 and the data processing in Sect. 3, I present the results in Sects. 4 and 5. First I address the question of the variation of the fractal dimension for two populations of active regions with different maximum magnetic fields obtained by Meunier (2003) and the variation of the fractal dimension during solar
Table 1. Average Wolf number for the 3 periods defined in the text.

\begin{tabular}{cc}
\hline \hline Period & Average Wolf number \\
\hline Cycle minimum & 10.3 \\
Ascending phase & 57.1 \\
Cycle maximum & 113.4 \\
\hline
\end{tabular}

flares. Then I consider the solar cycle variations in Sect. 5. The results are discussed in Sect. 6.

\section{Observations}

\subsection{MDI/SOHO: Full-disk magnetograms}

MDI/SOHO observations (Scherrer et al. 1995) provide maps of the LOS component of the magnetic field averaged over the resolution element ( 2 arcsec in the full disk mode). The observations are made in the Ni I $6768 \AA$ spectral line. I select 5-min full-disk magnetograms only, because they are less noisy (noise level of $\sim 8 \mathrm{G}$, see for example Meunier 1999a). This led to a data set of 7184 images between 23 May 1996 and 19 June 2002, covering 6 years. These magnetograms are separated by multiples of $96 \mathrm{~min}$. This data set is the one used in Meunier (2003).

To study solar cycle variations, I define the three following periods: the cycle minimum from day 143 (23 May 1996) until day 543 (27 June 1997), an ascending phase from day 543 until day 1243 (28 May 1999) and the cycle maximum after that date until the end of the data set. The corresponding average Wolf numbers are shown in Table 1 (see Meunier 2003, Fig. 1 for a plot of the Wolf number versus time for this period).

\subsection{The flare data set}

Flare data are obtained from the National Geophysical Data Center archive. Each $\mathrm{H} \alpha$ flare is counted once, and I extract from the data set the time, position and area of the flare. The number of $\mathrm{H} \alpha$ flares in the data set available during the period is 16140 . There are a few gaps in the flare data set, due to the reduced number of stations operating the flare patrol. However, in our analysis, the starting point is this flare data set, and for each flare we search for magnetograms observed around the same time: therefore, the relevant temporal coverage to consider is that of the magnetogram data set with respect to these flares. It was possible to select 7629 flares for which at least one magnetogram within $6 \mathrm{~h}$ of the flare exists. For a quarter of them, there is only one magnetogram available, while there are at least 2 (and up to 8) magnetograms for the other flares. Their average lifetime is $19 \mathrm{~min}$, and $83 \%$ of the flares have a lifetime shorter than $30 \mathrm{~min}$. 


\section{Data processing}

\subsection{Magnetogram processing}

\subsubsection{Region identification}

Magnetograms are analyzed using a method similar to that described in Meunier (2003). This is close to what was done in Meunier (1999a), except that we consider $B / \mu$ (where $\mu=$ $\cos \theta, \theta$ being the angle from disk center and $B$ is the LOS magnetic field provided by MDI) instead of $B$, because the magnetic field lines in both active regions and network regions are believed to be close to the vertical, within $10^{\circ 1}$. As in Meunier (2003), each structure above a given magnetic threshold (applied to the signed magnetograms) is determined within $53^{\circ}$ from disk center $\left(r<0.8 R_{\odot}\right)$. The area $A$ is defined by the number of pixels in the structure. The perimeter $P$ is derived from the pixel sides for which there is no neighbor. Areas and perimeters are corrected for projection effects and are converted to $\mathrm{Mm}^{2}$ and to $\mathrm{Mm}$ respectively. For each structure we also consider the average magnetic field $\bar{B}$ in that region as well as the maximum magnetic field $\left|B_{\mathrm{m}}\right|$ (both are fluxes averaged over the resolution element) and the flux $\Phi$ in the structure. Magnetic fields in this paper always mean magnetic fluxes (averaged over the resolution element) and not the actual field strength.

In this paper we mostly use a magnetic threshold of $40 \mathrm{G}$, which corresponds to 5 times the noise level on these magnetograms. The other thresholds (from $80 \mathrm{G}$ to $240 \mathrm{G}$ ) allow some comparisons with the results obtained earlier by Meunier (1999a) on a much smaller data set. The size distributions for the 3 periods of the solar cycle are shown and discussed in Meunier (2003, Fig. 7).

This method allows us to select magnetic structures corresponding to a given polarity, i.e. rather smaller scale individual structures of all sizes. Therefore they do not provide full active regions with both polarities. This allows us to make comparisons with previous results. However, when looking at the variation of the complexity of the structures with solar flares, this means that the complexity at this small scale is considered and not at the scale of the full region. This will be discussed in more detail in Sect. 4.2.

\subsubsection{Computation of the fractal dimension: Perimeter-Area method}

From the data set of structures defined in Sect 3.1.1, we define the fractal dimension $d$ from the relation between area and perimeter as:

$d=2 \frac{\Delta \log P}{\Delta \log A}$

which corresponds to twice the slope of the curve $P$ versus $A$ on a $\log / \log$ scale. If regions were perfect circles, or squares, then $d$ would be equal to unity. The more denticulate the structure, the closer $d$ is to 2 . This definition is very convenient and

1 This is not strictly the case in the penumbra. However, a similar analysis in which $B$ is used instead of $B / \mu$ leads to very similar results. has been widely used. It is described in more detail in Meunier (1999a). In the following, the fractal dimension is computed either from average $P$ versus average $A$ (where the average $P$ is computed over a size range of $\Delta \log A=0.1$ ) over size ranges of $\Delta \log A=1.5$ or, when indicated, over all data points (or a subset) in a given size range.

The first method had proved to be useful in the past (Meunier 1999a) because the fractal dimension is observed to vary with size (i.e. the structures are not strictly self-similar) and therefore one should consider the fractal dimension computed over smaller ranges of size. This method is used in Sect. 5 to allow some comparisons with the previous results. However, when few data points are available or when one wants to compute the fractal dimension for small subsets (in particular in size) I have computed the slope over all the points of the subset and this method is used in most of the paper. Note that both methods are used in Sect. 5.2.1 and they give similar results.

It has already been pointed out that one should be careful about the influence of spatial resolution on fractal dimension computations. For example, Meunier (1999a) found different behaviors for the full-disk and high-resolution MDI magnetograms and pointed out that it is necessary to establish comparisons between observations and simulations in similar conditions. This has also been discussed in detail by Janssen et al. (2003). However, when studying data obtained in similar conditions, the observed variations are still significant even though the comparison with other results (either observations or simulations) remains sometimes difficult.

\subsection{Association of the flares with a magnetic structure}

From the list of 85605 magnetic structures larger than $500 \mathrm{Mm}^{2}$ every $96 \mathrm{~min}$ or multiple of $96 \mathrm{~min}$ and the list of $\mathrm{H} \alpha$ flares, we need to associate each flare with an MDI structure observed close enough in time (before and after the flare). The parameters in this study are the following:

1. magnetic threshold on the magnetogram: $40 \mathrm{G}$;

2. minimum size for a magnetic structure: $500 \mathrm{Mm}^{2}$;

3. maximum distance $D_{\max }$ between the flare position and structure center of gravity: dependent on the size of the structure or fixed. Different values are studied. $D$ is computed after correction of the rotation rate for the different time of the flare and of the magnetogram;

4. maximum time lag between the MDI observation and the flare: $6 \mathrm{~h}$;

5. maximum variation of the size of the structure over the 12 h time lag: no limit or $40 \%$. The purpose of this parameter is to provide a constraint for a better identification of the same structure over time.

The two first parameters are related to the way the list of magnetic structures is built. Parameters 3 to 5 are related to the way the association flare-structure is performed. The limitations of this approach will be discussed in Sect. 4.2.2. 

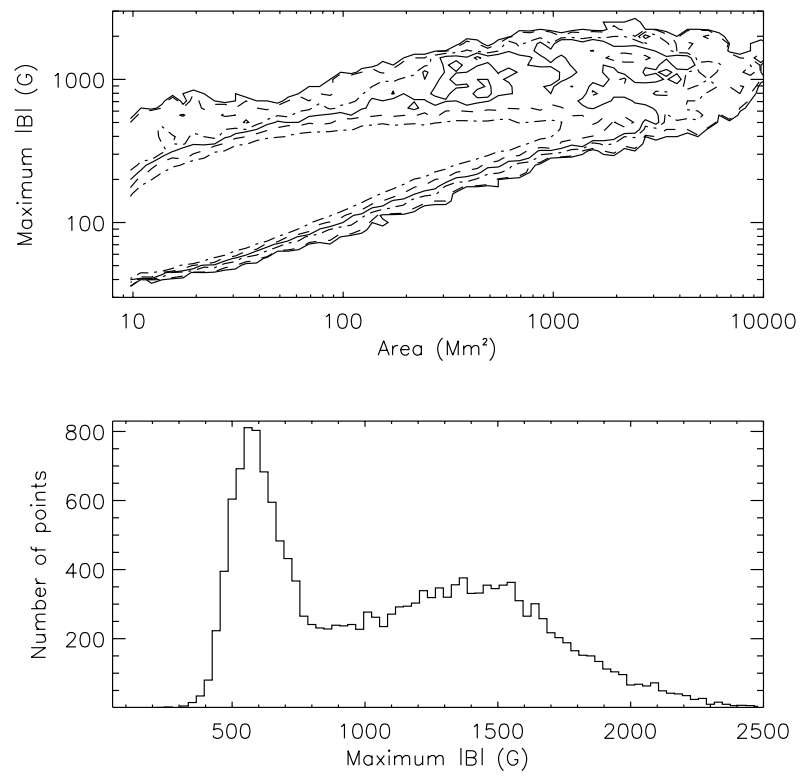

Fig. 1. Top: number of structures for each size and $\left|B_{\mathrm{m}}\right|$ ranges, for the whole period. The thickest lines correspond to thresholds of 100, 40, and 20 structures (respectively dotted-dashed, dashed, and solid). The thin lines correspond to thresholds of 10, 2, and 1 structures (respectively dotted-dashed, dashed, and solid). Bottom: number of structures versus $\left|B_{\mathrm{m}}\right|$, in the size range $2000-7000 \mathrm{Mm}^{2}$.

\section{Fractal dimension and activity of the region}

\subsection{Complexity and magnetic flux}

The 2-D distribution of the unsigned maximum magnetic field, $\left|B_{\mathrm{m}}\right|$, versus the size of the structures was shown by Meunier (2003) to exhibit a strong correlation between $\left|B_{\mathrm{m}}\right|$ and the size (Fig. 1, upper panel, for a threshold of $40 \mathrm{G}$ ). The distribution of $\left|B_{\mathrm{m}}\right|$ is however slightly more complex than that of $\bar{B}$ for example, because it exhibits a tail toward large values of $\left|B_{\mathrm{m}}\right|$ (especially for large sizes) corresponding to a second distribution (see lower panel in Fig. 1). In the range 2000-7000 $\mathrm{Mm}^{2}$ for example, where the phenomenon is the most visible, a narrow distribution peaks at $\sim 600 \mathrm{G}$ while the second one, broader, peaks at $\sim 1500 \mathrm{G}$. This 2 -component distribution is mostly present during the ascending phase and at solar maximum. In the range $2000-7000 \mathrm{Mm}^{2}$, structures with $\left|B_{\mathrm{m}}\right|$ larger than $900 \mathrm{G}$ (distribution peaking at $1500 \mathrm{G}$ ) have larger magnetic fluxes (by 35\%), larger flux density (by 27\%) and a larger ratio $B_{\mathrm{m}} / \bar{B}$ (by $22 \%$ ) than structures with a maximum magnetic field below $900 \mathrm{G}$. A given region is in the same category during all its lifetime. Although there are sunspots in both categories, most of the regions with spots are in the large $\left|B_{\mathrm{m}}\right|$ category while very few structures of the small $\left|B_{\mathrm{m}}\right|$ category have spots, as we checked visually from a subset of the data: it is plausible that larger $\left|B_{\mathrm{m}}\right|$ structures correspond to spots. Therefore the 2-component distribution is probably due to the presence or absence of spots in structures of a given size.

It is possible that the peak around $1500 \mathrm{G}$ could be an artifact due to the non-linearity in MDI LOS magnetic fields studied by Berger \& Lites (2003): this could lead to an excess of structures around the saturation magnetic field. This does not
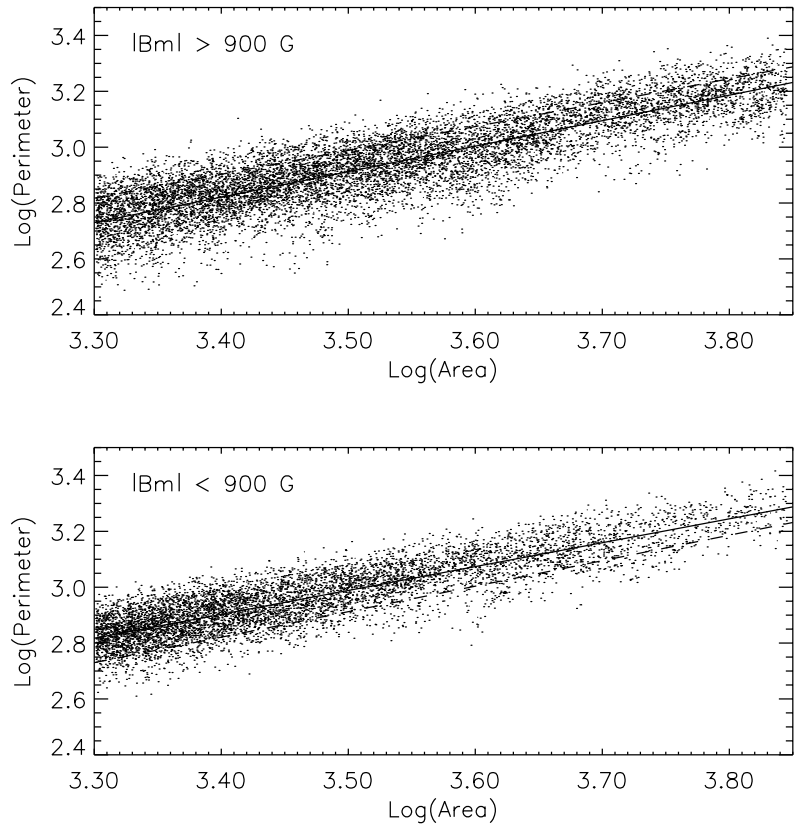

Fig. 2. Top: $\log$ of the perimeter $(\mathrm{Mm})$ versus $\log$ of the area $\left(\mathrm{Mm}^{2}\right)$ in the size range $2000-7000 \mathrm{Mm}^{2}$, for $\left|B_{\mathrm{m}}\right|>900 \mathrm{G}$. The solid line is a linear fit to the points, while the dashed line is a linear fit to the points for $\left|B_{\mathrm{m}}\right|<900 \mathrm{G}$ for comparison. Bottom: Log of the perimeter (Mm) versus $\log$ of the area $\left(\mathrm{Mm}^{2}\right)$ in the size range $2000-7000 \mathrm{Mm}^{2}$, for $\left|B_{\mathrm{m}}\right|<900 \mathrm{G}$. The solid line is a linear fit to the points, while the dashed line is a linear fit to the points for $\left|B_{\mathrm{m}}\right|>900 \mathrm{G}$ for comparison.

seem to be the case however, for two reasons: first, on the original values of $B$ (i.e. before the division of $B$ by $\mu$, which are the values to consider to compare with the Berger \& Lites results), the peak is visible around $1000 \mathrm{G}$, i.e. below the saturation level described by Berger \& Lites, i.e. $1300 \mathrm{G}$. Second, this peak is less pronounced on these data than on the $B / \mu$ data: this probably means that the peak is not due to an observational bias such as magnetic field saturation but is an intrinsic property of magnetic structures.

The fractal dimension in the range $2000-7000 \mathrm{Mm}^{2}$ is computed separately for the two categories (using a linear fit over all points of the selection). The fits are shown in Fig. 2. In this size range, structures with $\left|B_{\mathrm{m}}\right|$ larger than $900 \mathrm{G}$ have a fractal dimension of $1.82 \pm 0.01$, while structures with $\left|B_{\mathrm{m}}\right|$ smaller than $900 \mathrm{G}$ have a fractal dimension of $1.71 \pm 0.01$, which is significantly lower. The errorbars corresponds to the formal uncertainties on the fit and not the standard deviation of the fractal dimension of the individual structures, because with this method the fractal dimension can be computed only on a sample of structures, not on individual structures. This shows that structures of a given size with spots are more complex than structures of the same size with no spots. When looking at a larger domain, I find a similar result: structures with a larger $\left|B_{\mathrm{m}}\right|$ still seem to be more complex.

Because the structures are studied via their contours at low magnetic thresholds, the presence of sunspots inside the structures cannot bias the computations of their fractal dimension. Therefore, the difference in complexity must be due to the processes associated with the presence of spots, either during their 

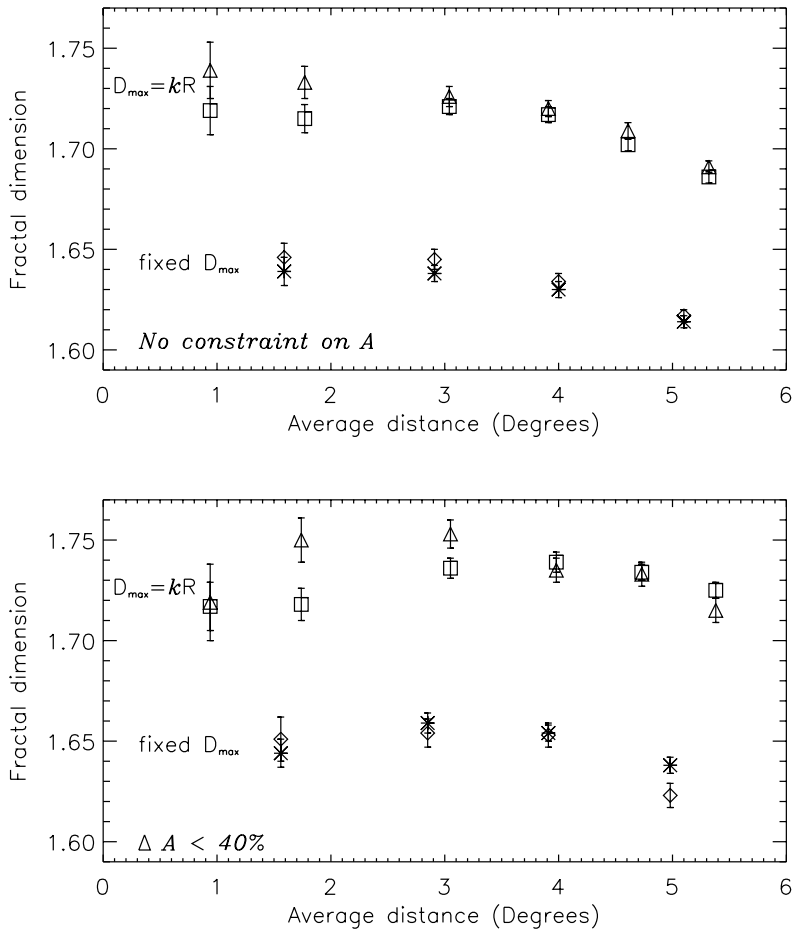

Fig. 3. Fractal dimension before (star) and after (diamond) the flares for fixed $D_{\max }$, as a function of the average distance $\langle D\rangle$ between the flare and the region. The squares and triangles represents the same fractal dimensions, but for $D_{\max }=k R$ (see text). Top: for no constraint on the size variation before and after the flares. Bottom: maximum variation of $40 \%$ of the size (see parameter \#5 in Sect. 3.2).

formation and emergence from the convective zone up to the photosphere, or during their evolution at the solar surface, in particular their dilution.

\subsection{Flare dependence}

\subsubsection{Results}

The comparison of the fractal dimension, averaged over $6 \mathrm{~h}$, before and after the flares is summarized in Fig. 3 for various average distances $D$ between the flare and the center of gravity of the magnetic structure. The maximum distance $D_{\max }$ between the flare location and the center of gravity of the structure (parameter \#3 in Sect. 3.2) is either constant or size-dependent ( $D_{\max }=k R$, with $\left.R=\sqrt{A / \pi}\right)$ and both possibilities are shown on each panel. The influence of a constraint on $A$ (parameter \#5 in Sect. 3.2) is also shown: no constraint for the top panel and a constraint on the bottom panel.

For most selections (i.e. when searching for structures at various distances from the flares and for various constraints on $A$ ), the fractal dimension is not significantly different before and after the flare. However, we observe a larger fractal dimension after the flare (significant at the 1- $\sigma$ level) in 3 cases, all for medium size $D_{\max }\left(\langle D\rangle\right.$ in the range $\left.1.5-3^{\circ}\right): D_{\max }=1 R$ and all $\Delta A, D_{\max }=1 R$ and $\Delta A<40 \%, D_{\max }=2 R$ and $\Delta A<40 \%$. We conclude than on average, the magnetic structure seems more complex after the flare when considering structures in the range $1.5-3^{\circ}$ from the flare, but only when the maximum
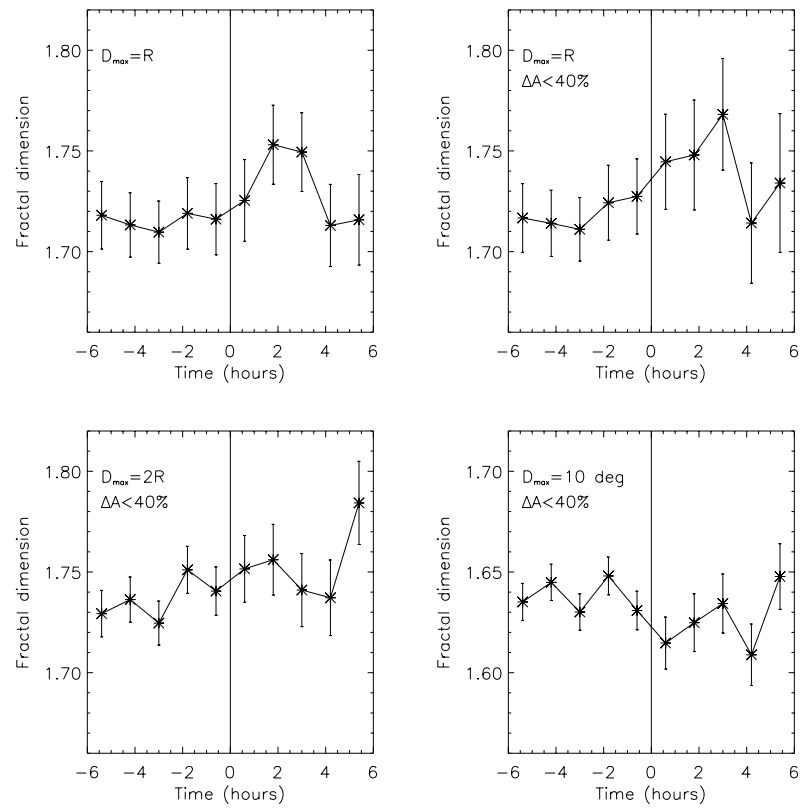

Fig. 4. Fractal dimension versus time for various values of $D_{\max }$. The vertical lines indicate the time of the flare: negative time correspond to the time before the beginning of the $\mathrm{H} \alpha$ flare, and positive correspond to the time after the end of the $\mathrm{H} \alpha$ flare.

distance is dependent on the size of the structure. Above a distance of $3^{\circ}$, and although the error bars are decreasing, no variation is observed except for one point $\left(D_{\max }=10^{\circ},\langle D\rangle=5.0^{\circ}\right.$, $\Delta A<40 \%$ ): this may be an indication that magnetic structures are less complex after the flare when including structures much further from the flare in the data set.

The same data set allowed us to look at the variation of the flux content of the structure before and after the flare. No significant variations of $\left|B_{\mathrm{m}}\right|, \bar{B}$ nor $\Phi$ are observed.

Figure 3 also shows that the fractal dimension is significantly larger $(\sim 0.1)$ when considering a variable $D_{\max }$. A detailed comparison of the set of structures selected for $D_{\max }=$ $7.5^{\circ}$ and $3 R$ (both have the same $\langle D\rangle$ ) shows that: 1) there is a small deficit of large structures and an excess of small structures in the $7.5^{\circ}$ subset, which would lead to smaller fractal dimension (see Sect. 5.1); 2) all sizes considered, the low $\left|B_{\mathrm{m}}\right|$ population (below $900 \mathrm{G}$ ) is twice the size for $D_{\max }=7.5^{\circ}$, and we have seen that this population is associated with a much smaller fractal dimension (see Sect. 4.1). These two effects are likely to explain the smaller fractal dimension obtained for fixed $D_{\max }$.

Figure 4 shows the variation of the fractal dimension with time, for the 4 cases mentioned above exhibiting a significant variation before and after the flares when averaged over $6 \mathrm{~h}$. The errorbars correspond to the formal errors of the linear fits for each subset of selected structures. With this better temporal resolution, no clear fluctuations are observed to occur within the error bars on short time scales. Furthermore, a subset of the flare data set (representing 35\% of the whole data set) is constructed by selecting only flares with no other flare occuring in the same region within 6 hours (defined from the NOAA number associated to the flare in the $\mathrm{H} \alpha$ flare list), to investigate the limitation pointed out in Sect. 3.2: with this selection, 
the results are noisier (due to the smaller number of points) and no obvious trend is observed.

As noted in Sect. 3.1.1, the structure identification is made using a threshold applied to structures of a given polarity. The study of the association of magnetic structures inside active regions is beyond the scope of this paper and will constitute a future work. However it was interesting to check whether the above results were confirmed and not biased by the fact that only sub-structures in the active regions were considered. An attempt was made to consider full active regions by associating the various magnetic structures of the data set using a closest neighbor analysis. With a definition of clusters representing active regions based on a maximum distance of $3^{\circ}$ between structures above $200 \mathrm{Mm}^{2}$, one can use the approach described above to associate a flare with these newly defined active regions. In that case, the size $R$ of the region used to defined $D_{\max }=k R$ is not based on the area but on the extension of the magnetic structures constituting the regions, and the limitation using $D_{\max }$ means that the flare occurs closer to the center of the active region or further away. With that definition of an active region, the results show that the fractal dimension decreases for a fixed $D_{\max }$ and the constraint of $40 \%$ on the size variation, either for small distance to the center of gravity of the region (lower than $2^{\circ}$ ) or much further (larger than $5^{\circ}$ ), but not in between. At large distances this is consistant with the previous result. At small distances, this is quite different, and it may imply that the active region as a whole has a behavior different from that of the individual magnetic structures close to a flare inside the active region.

Finally, we have attempted to consider subsets of the whole data set to check whether the amplitude of the variation was larger when only bright flares were involved. Unfortunately, although the large data set covers almost a full cycle, it is impossible to detect any variation because the error bars are several times larger than the observed variations for the whole data set.

\subsubsection{Limitations}

The limitations of this analysis are the following:

(i) Consider the variation of the fractal dimension with time before and after a given flare, i.e. the variations with $t=$ $t_{\text {flare }}-t_{\text {magnetogram }}$; all the variations are then then averaged together. Several flares can occur in the same area (parameter \#3) within $6 \mathrm{~h}$ (parameter \#4) ; for example, consider 2 flares (\#1 then \#2) separated by $2 \mathrm{~h}$. If there is an actual variation of the fractal dimension at the time of the flare, then this variation will appear not only at $t=0$ but also at $t=-2 h$ (due to flare \#1 when making the computation for flare \#2) and at $t=2 h$ (due to flare \#2 when making the computation for flare \#1). I have estimated that $65 \%$ of the flares of the whole data set corresponds to the same magnetic structure as at least another flare within $6 \mathrm{~h}$. Therefore the variation of the fractal dimension with time before and after flares may be smoothed, and this is probably the most serious limitation of the approach. It may explain partially the behavior of the curves of Fig. 4.

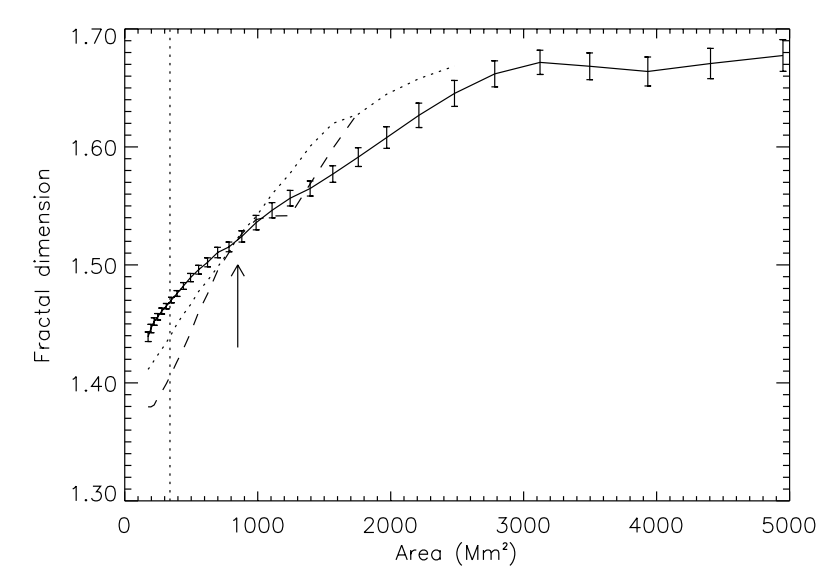

Fig. 5. Fractal dimension versus size for the whole periods. Solid line corresponds to $40 \mathrm{G}$, dotted to $80 \mathrm{G}$, dashed to $120 \mathrm{G}$ on all plots. For clarity, error bars are shown for the $40 \mathrm{G}$ threshold only. The arrows indicate the critical sizes discussed in the text. The vertical dotted line indicates the minimum size above which the fractal dimension computation does not include feature below 30 pixels.

(ii) Since we are looking at the magnetic structures within a certain distance of the flare, there may be several structures corresponding to the above criteria, therefore there may be a contribution from structures not directly involved in the flare despite their proximity to the flare; the actual structure at the origin of the flare could also be a small structure below the $500 \mathrm{Mm}^{2}$ threshold for example despite the presence of a larger region in the vicinity. This uncertainty will increase with increasing $D_{\max }$ and this should also smooth the signal. Studies of different values of $D_{\max }$ should help to bypass this problem, as studied from Fig. 3.

(iii) For a given flare, there may be magnetograms available before the flare but not after it, however these data will still be used. If the data sample is large enough, the result should not be biased although it will increase the noise.

\section{Fractal dimension over the solar cycle}

\subsection{Average fractal dimension}

Meunier (1999a) performed the fractal analysis of magnetic structures using MDI magnetograms (both 1 and 5 min images) until day 765 (4 February 1998), which corresponds to the solar minimum and the beginning of the ascending phase as defined here. The results for the whole period are shown in Fig. 5 for a magnetic threshold up to $120 \mathrm{G}$, for 5-min magnetograms only. For larger thresholds up to $240 \mathrm{G}$ I find results similar to that of Meunier (1999a) and they are not displayed here.

With the much longer data set studied here, I also find that the fractal dimension, although varying with the size of the structure (as in Meunier 1999a), reaches a plateau above $3000 \mathrm{Mm}^{2}$ (linear size of $55000 \mathrm{~km}$ ) with an average value of 1.67 .

Meunier (1999a) also found a critical size around $600 \mathrm{Mm}^{2}$ : this size corresponded to a "pivot point" between large structures, for which the fractal dimension was increasing for increasing magnetic thresholds, and smaller structures, 

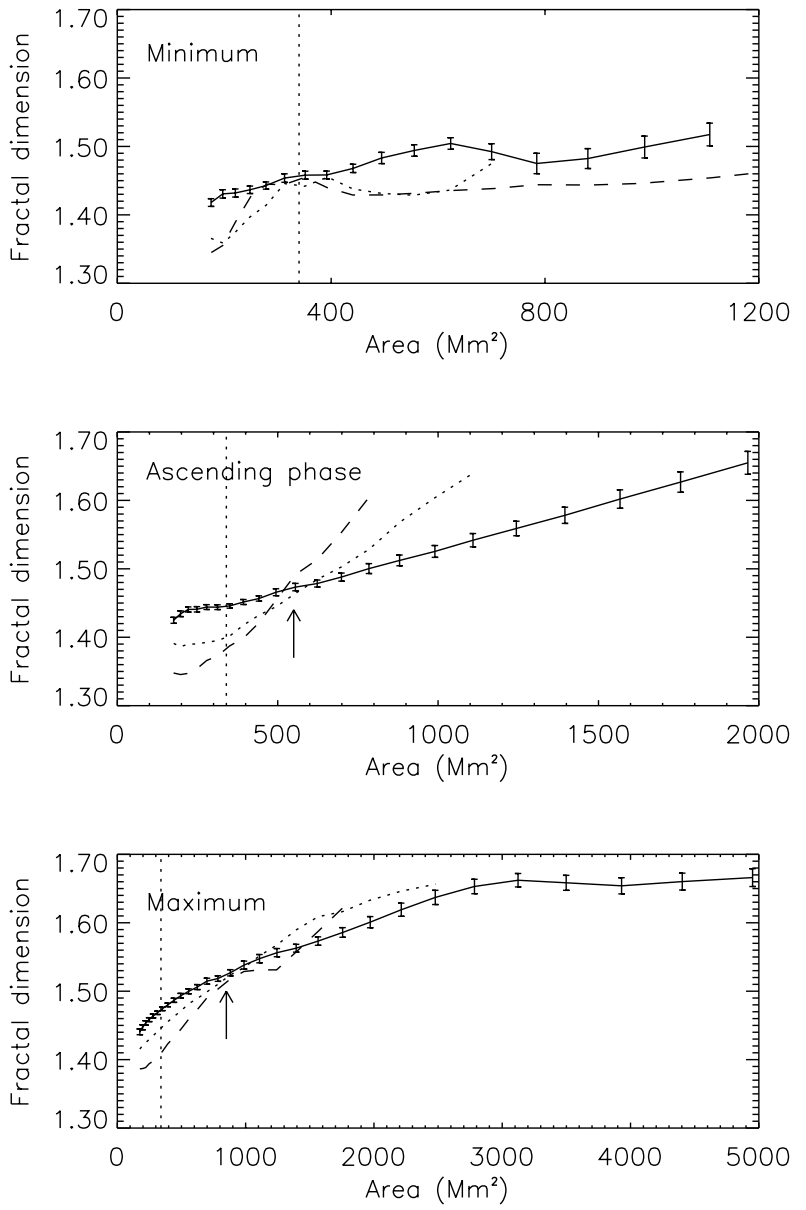

Fig. 6. Fractal dimension versus size for the 3 periods of the solar cycle, on the same vertical scale. Solid line corresponds to $40 \mathrm{G}$, dotted to $80 \mathrm{G}$, dashed to $120 \mathrm{G}$ on all plots. For clarity, error bars are shown for the $40 \mathrm{G}$ threshold only. The arrows indicate the critical sizes discussed in the text. The vertical dotted line indicates the minimum size above which the fractal dimension computation does not include feature below 30 pixels and is therefore reliable.

for which the fractal dimension was decreasing. Here I confirm this on a much larger data set: the position of this critical point is shown by an arrow in Fig. 5. One should remember that a given structure detected at a different magnetic threshold corresponds to a different size in these plots. The origin of such a "pivot point" is not well understood: The "pivot point" is not only due to the fact that for increasing threshold (i.e. field closer to the core of the region), the structures are less and less "fractal" (dimension varies more strongly with size), but also that they happen to cross at the same scale. Models should be able to reproduce this type of results to be realistic.

\subsection{Cycle phase dependence}

\subsubsection{Influence of the size}

Figure 6 shows the variation of the fractal dimension with size for the 3 phases of the cycle defined in Sect. 2. The critical size has a value of $\sim 550 \mathrm{Mm}^{2}$ during the ascending phase

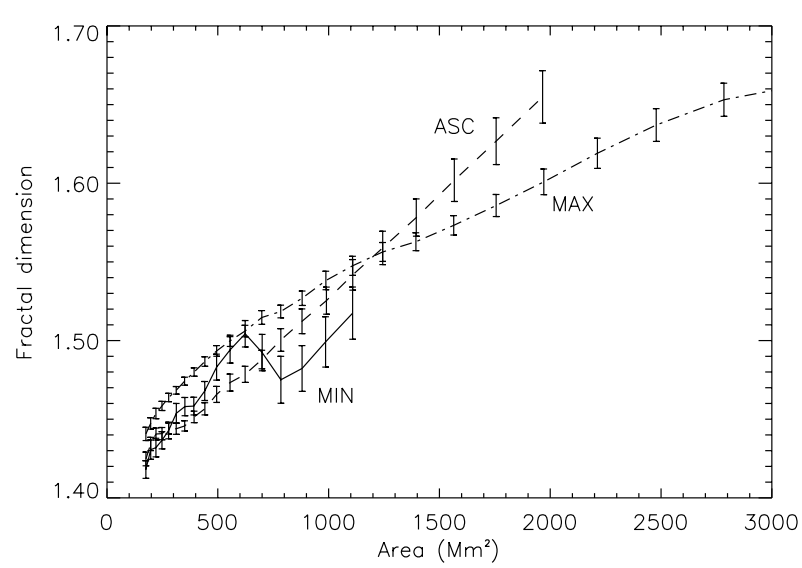

Fig. 7. Fractal dimension versus size for the 3 periods at the $40 \mathrm{G}$ threshold. Solid line corresponds to cycle minimum, dashed line to the ascending phase and the dotted-dashed line to cycle maximum.

(which is similar to the result of Meunier 1999a) and of $\sim 850 \mathrm{Mm}^{2}$ during cycle maximum.

A comparison of the variations during the solar cycle for the $40 \mathrm{G}$ threshold is shown in Fig. 7. The complex pattern is similar when observed at the $80 \mathrm{G}$ threshold (although shifted toward smaller sizes of course). At the $40 \mathrm{G}$ threshold, structures larger than $1200 \mathrm{Mm}^{2}$ are more complex during the ascending phase than during the cycle maximum (there are very few such structures during cycle minimum). Between the ascending phase and cycle maximum, the variation reaches 0.06 when considering the largest structures. Below this size down to $A \sim 700 \mathrm{Mm}^{2}$ (i.e. close to the "pivot point" mentioned above), I observe the opposite, with structures less complex during solar minimum: in this range, structures are more complex when the activity level is larger. For even smaller structures, the fractal dimension at cycle minimum is intermediate between the other curves. In the range where the fit is significant (i.e. including size above 30 pixels only as shown in Meunier 1999a, or $60 \mathrm{Mm}^{2}$ ), the difference of fractal dimension between cycle maximum and minimum is of the order of 0.02 only (this is for small structures since there are almost no large structures during solar minimum), the structures at cycle maximum beeing only slightly more complex than at cycle minimum (this is marginally significant).

Another way to look at this is to compute directly the slope of $\log (P)$ versus $\log (A)$ for all points in a given size range. For example, for $A$ in the range $800-1200 \mathrm{Mm}^{2}$, the fractal dimension is $1.19 \pm 0.17$ at cycle minimum, $1.38 \pm 0.07$ during the ascending phase and $1.47 \pm 0.03$ during the maximum, showing a significant increase from cycle minimum to cycle maximum. On the other hand, for $A>1200 \mathrm{Mm}^{2}$, the fractal dimension is $1.59 \pm 0.04$ at cycle minimum, $1.78 \pm 0.01$ during the ascending phase and $1.669 \pm 0.004$ during the maximum, exhibiting the largest value during the ascending phase, the fractal dimension remaining the smallest during cycle minimum. This confirms the results shown in Fig. 7. 
Table 2. Fractal dimension and number of points (in italic) in the size range 2000-7000 $\mathrm{Mm}^{2}$.

\begin{tabular}{ccc}
\hline \hline Period & $\left|B_{\mathrm{m}}\right|<900 \mathrm{G}$ & $\left|B_{\mathrm{m}}\right|>900 \mathrm{G}$ \\
\hline Total & $1.71 \pm 0.01(10147)$ & $1.89 \pm 0.01(7273)$ \\
Cycle minimum & $1.09 \pm 0.11(2432)$ & $1.53 \pm 0.06(143)$ \\
Ascending phase & $1.64 \pm 0.03(1047)$ & $1.97 \pm 0.03(1385)$ \\
Cycle maximum & $1.73 \pm 0.01(6097)$ & $1.80 \pm 0.01(8619)$ \\
\hline
\end{tabular}

\subsubsection{Influence of $B_{\mathrm{m}}$}

When one separates the 2 populations described in Sect. 4.1 (with low $\left|B_{\mathrm{m}}\right|$ and large $\left|B_{\mathrm{m}}\right|$ ), one obtains the results shown in Table 2 for the size range $2000-7000 \mathrm{Mm}^{2}$ : from the ascending phase to cycle maximum, a strong increase $(+0.09)$ is observed for low $\left|B_{\mathrm{m}}\right|$ structures, while a strong decrease $(-0.17)$ is observed for strong $\left|B_{\mathrm{m}}\right|$ structures. This also means that the difference between the fractal dimensions of the 2 populations is larger during the ascending phase compared to cycle maximum. Note that although the proportion of strong $\left|B_{\mathrm{m}}\right|$ structures is slightly larger at cycle maximum, the effect on the fractal dimension is expected to be very small (0.001).

During cycle minimum, some of the largest regions are likely to be due to the end of the previous cycle, i.e. some of them may correspond to the end of a maximum phase rather than to the beginning of the studied cycle. Indeed, at cycle minimum, the latitude distribution is made up of two components, one centered on $7.5^{\circ}$ (68\% of the points, corresponding to the end of the previous cycle) and the other on $26.7^{\circ}$ (32\% of the points, corresponding to the beginning of the current cycle). This suggests that from the beginning of the ascending phase until the very end of maximum period, the very large active regions may be less and less complex. However there are few points during cycle minimum (1094 structures larger than $800 \mathrm{Mm}^{2}$ only).

The variations during the solar cycle for the different components is also shown in Fig. 8 with better temporal resolution. On this plot, the values for low latitudes at cycle minimum are shown at the end of the time series (see caption of Fig. 8). One can clearly see the very different behavior of larger and small structures and of different $\left|B_{\mathrm{m}}\right|$ structures for the largest sizes. Large structures with high $\left|B_{\mathrm{m}}\right|$ have their maximum fractal dimension during the middle of the ascending phase ( 2 years after the beginning of the activity raise), then they decrease to reach an approximately constant level during cycle maximum. A small increase is also observed at the end of cycle maximum. The structures of the same size with low $\left|B_{\mathrm{m}}\right|$ exhibits an increase of the fractal dimension during the ascending phase, a maximum during solar maximum and then a decrease and finally a small increase as for the large $\left|B_{\mathrm{m}}\right|$ structures. Small structures shows a behavior like the latter structures, also the detailed variations during cycle maximum may be slightly different. At the end of the cycle we expect to observe much smaller fractal dimensions for all structures.

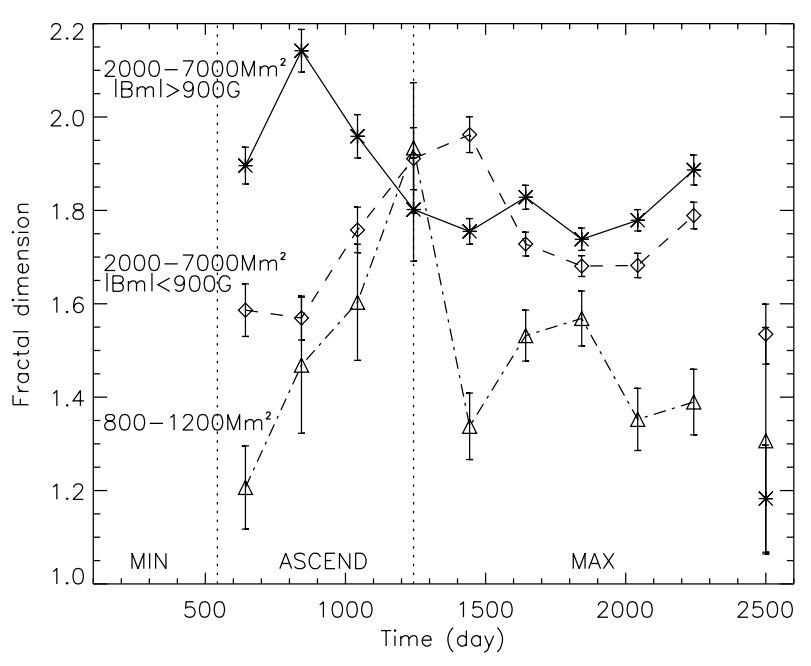

Fig. 8. Fractal dimension versus time for various selections: $A$ in the range $800-1200 \mathrm{Mm}^{2}$ (dotted-dashed line and triangles), $A$ in the range 2000-7000 $\mathrm{Mm}^{2}$ and $B_{\mathrm{m}}>900 \mathrm{G}$ (solid line and stars), and $A$ in the range 2000-7000 $\mathrm{Mm}^{2}$ and $B_{\mathrm{m}}<900 \mathrm{G}$ (dashed line and diamonds). The points at $t=2500$ days correspond to the structures of cycle minimum $\left(t<543\right.$ days) with latitude smaller than $17^{\circ}$, i.e. to the end of the previous cycle, and are shown arbitrarily at this time. The same structures for latitude larger than $17^{\circ}$ (i.e. beginning of the studied cycle) are not shown because of the very large errorbars (there are very few large structures during cycle minimum).

\subsubsection{Influence of latitude}

It is also useful to check whether the observed variations with time are due to latitude or to temporal variations. It is not easy to do since most structures are at different latitudes during the ascending phase and the cycle maximum. I have considered the latitude range $17-22^{\circ}$ in which there are many structures in both cases. When considering the size range 2000-7000 $\mathrm{Mm}^{2}$ studied above, a small increase of the fractal dimension is observed from the ascending phase to cycle maximum, but this is mostly due to the large increase for the $\left|B_{\mathrm{m}}\right|<900 \mathrm{G}$ structures, while there is still a decrease for the $\left|B_{\mathrm{m}}\right|>900 \mathrm{G}$ structures. When considering the range $A>1200 \mathrm{Mm}^{2}$, I still observe a decrease of half the size of that for all points (from $1.77 \pm 0.02$ to $1.72 \pm 0.01)$ while I observe an increase for the size range $800-1200 \mathrm{Mm}^{2}$ (from $1.25 \pm 0.13$ to $1.33 \pm 0.06$ ), although this is not significant due to the small number of points in that latitude range.

I deduce from these computations that the time variation is real, as are the different properties between the two populations observed in Sect. 4.1. Furthermore, this is reinforced by the complex evolution through the cycle observed in Fig. 8, which is not correlated at all with the also complex latitude variation and the variation that could be expected from it due to the butterfly diagram.

Finally, when considering the latitude distribution for $\left|B_{\mathrm{m}}\right|<900 \mathrm{G}$ and $\left|B_{\mathrm{m}}\right|>900 \mathrm{G}$ in the size range 2000-7000 $\mathrm{Mm}^{2}$, a small deficit of the latter is observed in the latitude range $20-30^{\circ}$. However the difference of fractal dimensions between the range $0-20^{\circ}$ and $20-30^{\circ}$ is too small 
to explain the behavior of these two populations observed in Sect. 4.1.

\section{Discussion}

I have performed several analyses to study the dependence of the fractal dimension of magnetic structures on the activity level at different time scales. The following results have been obtained:

(i) The variation of the fractal dimension of magnetic structures computed on LOS magnetograms due to flares is observed to be very small, and in most cases hardly significant, despite the very large sample compared to previous studies. Close to the flares $\left(2-3^{\circ}\right)$, an increase towards larger complexity of individual magnetic structures is observed. On the other hand, when considering large structures further from the flares (with distance up to $10^{\circ}$ ), there is a tendancy to get smaller fractal dimensions after the flare, i.e. less complex structures, suggesting largescale modifications as pointed out by Yurchyshyn et al. (2000). The very small variations could be due to the fact that there are other flares in the active region within the studied time interval, but a subset of flares that does not have this limitation does not show any significant trend with time either. Because the variations that are observed are very small and not very significant despite the large sample, it does not seem realistic to use the variation of the LOS magnetic fields to predict solar flares. This confirms previous results obtained by Adams et al. (1997) for example, but on a much larger sample. It could indeed be expected that most of the topology modifications should happen in that layer and not in the photosphere, as studied with the MDI magnetograms, since the energy release during a flare takes place mostly in the chromosphere.

(ii) Structures with spots (strong $\left|B_{\mathrm{m}}\right|$ ) are significantly more complex than structures with no spots $\left(\right.$ low $\left.\left|B_{\mathrm{m}}\right|\right)$, for a given size. This is surprising because we could expect that many large structures with no spots are decayed structures with an apparently complex structure (more diluted structure). The effect is more pronounced for large structures.

(iii) The general behavior of the fractal dimension (in particular the dependence on the size of the structures) obtained by Meunier (1999a) is confirmed on a much larger data sample, especially for large scales. The "pivot point", also observed by Meunier (1999a) and close to the supergranular size, is observed to increase from solar minimum to solar maximum. A plateau above $3000 \mathrm{Mm}^{2}$ is also observed, with an average fractal dimension of 1.67 .

(iv) Variations of the order of 0.02 to 0.06 (depending on the size) of the fractal dimension are observed during the cycle. The sign of the variation depends on the size range and on $\left|B_{\mathrm{m}}\right|$. The results are complicated by the different behavior of the strong $\left|B_{\mathrm{m}}\right|$ structures (which, on average, exhibit a decrease of the fractal dimension from the ascending phase to cycle maximum) and the low $\left|B_{\mathrm{m}}\right|$ structures (which shows an increase, as observed for medium size structures above $800 \mathrm{Mm}^{2}$ ). When one separates these populations, the amplitude of variations is of the order of $0.1-0.2$. Therefore structures with spots seem more complex at the beginning of the cycle compared to cycle maximum and the structures with no spots seem less complex. The detailed temporal variations are also quite complex, with the fractal dimension peaking at different times for the structures of different sizes or different $\left|B_{\mathrm{m}}\right|$ (ranging from the middle of the ascending phase to the beginning of cycle maximum). We showed that these variations are mostly due to a temporal variation and not to a latitudinal dependence.

The linear size derived from the square root of the structure areas shows that the "pivot point" at $\sim 800 \mathrm{Mm}^{2}$, which corresponds to $28300 \mathrm{~km}$, happens to be close to the supergranular scale. A scale of similar amplitude was also identified by Meunier (2003) from the size distribution of active regions. They could be related, since in both cases they seem to increase during the solar cycle. This particular pattern should be taken into account by models. The different complexity behavior for different sizes may be due to a different formation process for small and large active regions: I suggest that models looking at the formation of active regions such as those of Wentzel \& Seiden (1994), Seiden \& Wentzel (1996) and more recently of Vlahos et al. (2002) should try to reproduce this result.

The variation of the fractal dimension with the solar cycle is one of the most important results and models should also be able to reproduce this. Whether they are due to a variation of the dynamo process during the cycle (including a variation with depth) or to surface effects affecting the decay of active regions is still an open question. The magnetic field must play an important role given the large variation of the fractal dimension with the size of the structures and with $\left|B_{\mathrm{m}}\right|$. This should favor a dynamo origin. The non-monotonous variation, with a peak during the ascending phase or cycle maximum, could be associated with the influence of two processes that would be out of phase. On the other hand, surface flows may influence in different manners structures of different sizes. Harvey (1993) showed that during cycle maximum, structures have a longer lifetime than at cycle minimum, with a factor $\sim 1.3$ which is independent of the size for $A>1500 \mathrm{Mm}^{2}$ but this factor seems to be slightly smaller for smaller sizes. This could lead to a longer decay phase at cycle maximum that would lead to diluted structures a larger proportion of the time and therefore a larger fractal dimension at cycle maximum. This could not be the only factor however since the observed variations are much more complex than this. Divergent and convergent flows around active regions could also play a role. On a large scale, convergent flows are observed (Meunier 1999b; Chou \& Dai 2001; Beck et al. 2002) although on smaller scales the moat flow is divergent. The latter is likely to contribute to the erosion of active regions and to their dilution in a more complex pattern. The moat flows around sunspots may also be part of the reason why the structures with spots are more complex. A study allowing one to follow and separate young and old regions would also be useful to sort out the different contributions.

Finally, the observed timescale of a few years (peak of fractal dimension after the activity level has started to rise) leads to 
the question of whether this behavior is purely transient or related to some oscillatory behavior such as the 1.3 year period observed in the rotation rate at the bottom of the tachochline (Howe et al. 2000) or to the multipeaked structures observed in the Wolf number during the ascending phase and cycle maximum. More observations during future cycles will be necessary to investigate this in more detail.

Acknowledgements. SOHO is a mission of international cooperation between the European Space Agency (ESA) and NASA. Sunspot numbers and flare data have been provided by the National Geophysical Data Center (National Oceanic and Atmospheric Administration). I gratefully ackowledge the referee for his remarks which have helped to improve the paper.

\section{References}

Abramenko, V. I., Yurchyshyn, V. B., Wang, H., Spirock, T. J., \& Goode, P. R. 2002, ApJ, 577, 487

Adams, M., Hathaway, D. H., Stark, B. A., \& Musielak, Z. E. 1997, Sol. Phys., 174, 341

Aulanier, G., DeLuca, E. E., Antiochos, S. K., McMullen, R. A., \& Golub, L. 2000, ApJ, 540, 1126

Balke, A. C., Schrijver, C. J., Zwaan, C., \& Tarbell, T. D. 1993, Sol. Phys., 143, 215

Beck, J. G., Gizon, L., \& Duvall, T. L. 2002, ApJ, 575, L47

Bleybel, A., Amari, T., van Driel-Gesztelyi, L., \& Leka, K. D. 2002, A\&A, 395, 685

Chou, D. Y., \& Dai, D. C. 2001, ApJ, 559, 175

Fletcher, L., Metcalf, T. R., Alexander, D., Brown, D. S., \& Ryder, L. A. 2001, ApJ, 554, 451

Hagyard, M. J., Stark, B. A., \& Venkatakrishnan, P. 1999, Sol. Phys., 184,133
Harvey, K. L. 1993, Ph.D. Thesis, Astronomical Institute, Utrecht University

Howe, R., Christensen-Dalsgaard, J., Hill, F., et al. 2000, Science, 287(5462), 2456

Janssen, K., Vögler, A., \& Kneer, F. 2003, A\&A, 409, 1127

Nesme-Ribes, E., Meunier, N., \& Collin, B. 1996, A\&A, 308, 213

Mathew, S. K., \& Ambastha, A. 2000, Sol. Phys., 197, 75

Meunier, N. 1999a, ApJ, 515, 801

Meunier, N. 1999b, ApJ, 527, 967

Meunier, N. 2003, A\&A, 405, 1107

Patty, S. R., \& Hagyard, M. J. 1986, Sol. Phys., 103, 111

Ruzmaikin, A., Sokoloff, D., \& Tarbell, T. 1991, in The Sun and Cool Stars: Activity, Magnetism, Dynamos, ed. I. Tuominen, D. Moss, \& G. Rüdiger (Berlin: Springer), 140

Scherrer, P. H., Bogart, R. S., Bush, R. I., et al. 1995, Sol. Phys., 162, 129

Seiden, P. E., \& Wentzel, D. G. 1996, ApJ, 460, 522

Sorriso-Valvo, L., Carbone, V., Veltri, P., et al. 2003, Planet. Space Sci., in press

Stark, B., Adams, M., Hathaway, D. H., \& Hagyard, M. J. 1997, Sol. Phys., 174, 297

Tao, L., Du, Y., Rosner, R., \& Cattaneo, F. 1995, ApJ, 443, 434

Vainshtein, S. I., Sagdeev, R. Z., Rosner, R., \& Kim, E.-J. 1996, Phys. Rev. E, 53(5), 4729

Vlahos, L., Fragos, T., Isliker, H., \& Georgoulis, M. 2002, ApJ, 575, L87

Wentzel, D. G., \& Seiden, P. E. 1992, ApJ, 390, 280

Yurchyshyn, V. B., Abramenko, V. I., \& Carbone, V. 2000, ApJ, 538, 968

Zelenyi, L. M., \& Milovanov, A. V. 1991, Soviet Astron. Lett., 17(6), 425

Zhang, C., \& Wang, J. 2000, Sol. Phys., 196, 377

Zhang, C., \& Wang, J. 2002, Sol. Phys., 205, 303 